\title{
Optimal Routing Strategy Based on Specifying Shortest Path
}

\author{
F. Shao, B. Cheng
}

\author{
Fei Shao*, Binghua Cheng \\ School of Computer Engineering, Jinling Institute of Technology \\ No. 99, Hongjing Rd., Nanjing, 211169, JiangSu, P.R.China \\ *Corresponding author: shaofei@jit.edu.cn \\ cbh@jit.edu.cn
}

\begin{abstract}
How to enhance the transfer capacity of weighted networks is of great importance. The network transfer capacity, which is often evaluated by the critical packet generation rate, is proved to be inversely proportional to the highest node betweenness. By specifying the shortest path according to the different node characteristics, two different routing strategies are proposed to reduce the high node betweenness for the different node delivery capability schemes. Simulations on both computer-generated networks and real world networks show that our routing strategies can improve the network transfer capacity greatly. Especially, the greater the new added edge number is, the more efficient our routing strategies are.
\end{abstract}

Keywords: complex networks, weighted networks, routing strategy, betweenness.

\section{Introduction}

Due to the constantly growing significance of large scale communication networks such as the World Wide Web, the network transfer capacity has attracted an increasing attention. In those previous studies to improve transfer capacity and control traffic congestion on networks, some focus on making appropriate adjustments to the network topology structure [1] - [3] while others on finding optimal routing strategies [4] - [9]. Since the former is too expensive and too difficult to implement in some large-scale networks, most of previous works focused on effective routing strategies. Some of them are based on the global information: the shortest path routing strategy [4] which pass through the minimum number of nodes, the efficient path routing strategy [5] whose sum of node degrees is the minimum. Some others focus on local topological information since global information is usually unavailable in large-scale networks: the neighbor information [6], the next-nearest-neighbor information [7].

However, those aforementioned studies are mostly focused on the simplest unweighted networks with edges between nodes are represented by binary states according to whether the edges are present or not. In fact, the scientific collaboration networks [10], the cellular metabolism [11], the world-wide airport networks [12] and the Internet [13] have been proved to be weighted networks which are specified not only by its topology but also by the weight of the edges. Lots of models have been presented to describe weighted network among which the BBV weighted network model, coupled dynamical evolution of topology and weights, is most widely used.

In those most widely used traffic models in weighted network, the packets are transferred through the traditional shortest path [4], which is a path with the minimal number of nodes between arbitrary pairs of nodes, or the weighted shortest path $[10,14]$, where the distance between nodes is just the inverse of the weight of edge linked them. However, it is proved that these two routing strategies are not optimal for weighted networks [15]. Since there may be more than one traditional shortest path between some pairs of nodes, we can specify the best one to enhance the network transfer capacity.

This paper is organized as follows. In section 2 we describe the model and our routing strategy, followed by the experimental evaluations on the computer generated networks and real world network in section 3. The conclusions are given in section 4 . 


\section{Models}

\section{$2.1 \quad$ Network Model}

The BBV network can be completely described by a weighted adjacency matrix $W$ whose elements $w_{i j}$ denote the weight of the edge linking node $i$ and $j$. The generation of the BBV network is based on two coupled mechanisms:

i Topological growth. Starting from the initial network with $N_{0}$ nodes which are fully connected by edges with assigned weight $w_{0}$, one new node is added at every time step. The new added node will be connected to $m$ different nodes with equal weight $w_{0}$ for every edge and will choose nodes with large strength according to the probability $\prod_{n \rightarrow i}=s_{i} / \sum_{l} s_{l}$, where $s_{i}=\sum_{j} w_{i j}$ is the node strength.

ii Weight dynamics. The weight of each new added edge is initially set to a given value $w_{0}$ which is often set to 1 for simplicity. But the adding of edge connecting to node $i$ will result in increasing the weight of the other edges linked to node $i$ which is proportional to the edge weights. If the total increase is $\delta$ (we will focus on the simplest form: $\delta_{i}=\delta$ ), we can get

$$
w_{i j}=w_{i j}+\Delta w_{i j}=w_{i j}+\delta * \frac{w_{i j}}{s_{i}}
$$

This will yield the strength increase of node $i$ as:

$$
s_{i}=s_{i}+\delta+w_{0}
$$

The degree distribution of BBV network $P(k) \propto k^{-\gamma_{k}}$ and the strength distribution $P(s) \propto$ $s^{-\gamma_{s}}$ yield scale-free properties with the same exponent [12], [16]- [18]:

$$
\gamma_{k}=\gamma_{s}=\frac{4 \gamma+3}{2 \gamma+1}=2+\frac{1}{2 \gamma+1}
$$

\section{$2.2 \quad$ Traffic Model}

The traffic model can be described as follows:

i All nodes can create packets with addresses of destination, receive packets from other nodes, and forward the packets to their destinations.

ii At each time step, there are $R$ packets generated in the network, with randomly chosen sources and destinations. Once a packet is created, it is placed at the end of the queue if the node already has several packets waiting to be forwarded to their destinations.

iii At each time step, the first $C_{i}$ packets at the top of the queue of node $i$, if it has more than $C_{i}$ packets in its queue, are forwarded one step toward their destinations and placed at the end of the queues of the selected nodes. Otherwise, all packets in the queue are forwarded one step. This procedure applies to all nodes at every time step.

iv A packet, upon reaching its destination, is removed from the system. 
In our model, three node delivery capability schemes are considered: (i) each node has the same packet delivery capability $\left(C_{i}=1\right.$, CON stands for this scheme); (ii) the node delivery capacity is considered to be proportional to the node strength $s_{i}\left(C_{i}=s_{i} /\langle s\rangle\right.$, STR stands for this scheme); (iii) the node delivery capacity is considered to be proportional to the node degree $k_{i}\left(C_{i}=k_{i} /<k>\right.$, DEG stands for this scheme). To compare the overall transfer capacity, we normalize the delivery capability to keep the total node delivery capability of the whole network is equal to the node number $n$ in three situations the same. When $R$ is increased from 0 to $\infty$, two phases will be observed: free flow and congested phase. For $R<R_{c}$, the numbers of created and forwarded packets are balanced, resulting in a steady free flow of traffic. For $R>R_{c}$, traffic congestion occurs due to the fact that packet delivery capacity of node is limited. The phase transition from the former to the latter occurred at the critical packet generation rate $R_{c}$. We focus on the critical value $R_{c}$ which can best reflect the transfer capacity of a network.

We utilize the betweenness $b_{i}[19]$ to estimate the traffic passing through a node $i$ under a given routing strategy:

$$
b_{i}=\sum_{s, t} \frac{\sigma(s, i, t)}{\sigma(s, t)}
$$

where $\sigma(s, i, t)$ is the number of paths under the given routing strategy between nodes $s$ and $t$ that pass through node $i$ and $\sigma(s, t)$ is the total number of paths under the given routing strategy between $s$ and $t$ and the sum is over all pairs $s, t$ of all distinct nodes.

The probability a packet will pass through the node $i$ is $b_{i} / \sum_{j=1}^{n} b_{j}$, and therefore the average number of packets that the node $i$ will receive at each time step is. When the number of incoming packets is equal to or larger than the outgoing packets at the node $i, R b_{i} /(n(n-1)) \geq C_{i}$, traffic congestion will occur. So the critical packet generation rate $R_{c}$ is

$$
R_{c}=\min \left(\frac{C_{i} * n *(n-1)}{b_{i}}\right)=n *(n-1) * \min \left(C_{i} / b_{i}\right)
$$

\section{$2.3 \quad$ Routing Strategy}

Enlightened by the efficient path [5], we also define $P_{i \rightarrow j}$ as the path between node $i$ and $j$ which pass through the nodes sequence $x_{0}(=i), x_{1}, x_{2}, \cdots, x_{n-1}, x_{n}(=j)$. However we define

$$
F\left(P_{i \rightarrow j}, \alpha\right)=\sum_{i=0}^{n-1} w_{i j}^{\alpha}
$$

In our routing strategies, we specify the path between $i$ and $j$ as the one makes $F\left(P_{i \rightarrow j}, \alpha\right)$ minimum under a given tunable parameter $\alpha$. When $\alpha$ is -1 , the specified routing strategy is the same as the weighted shortest path routing strategy [10,14] (WSH stands for this routing strategy). When $\alpha$ is 0 , the specified routing strategy is the same as the traditional dijkstra shortest path routing strategy [4] which pass through the minimum amount of nodes (SHT stands for this routing strategy). As we mentioned above, there may be more than one shortest path between some nodes. We calculate the sum of strengths $s_{i}$ of all nodes on each shortest path, and select the one with minimum sum as our specified path. SSS stands for this routing strategy and SSD for the minimum sum of degrees $k_{i}$. The definition of our routing strategies (the SSS routing strategy and the SSD routing strategy) is shown in Tab.1. 
Table 1: Definition of our routing strategies (SSS and SSD)

\begin{tabular}{|c|c|c|c|c|}
\hline & WSH & SHT & SSS & SSD \\
\hline$F\left(P_{i \rightarrow j}, \alpha\right)$ & $\min \left(\sum_{i=0}^{n-1} w_{i j}^{-1}\right)$ & $\min \left(\sum_{i=0}^{n-1} w_{i j}^{0}\right)$ & $\operatorname{SHT\& min}\left(\sum_{i=0}^{n-1} s_{i}\right)$ & $\operatorname{SHT\& min}\left(\sum_{i=0}^{n-1} k_{i}\right)$ \\
\hline
\end{tabular}

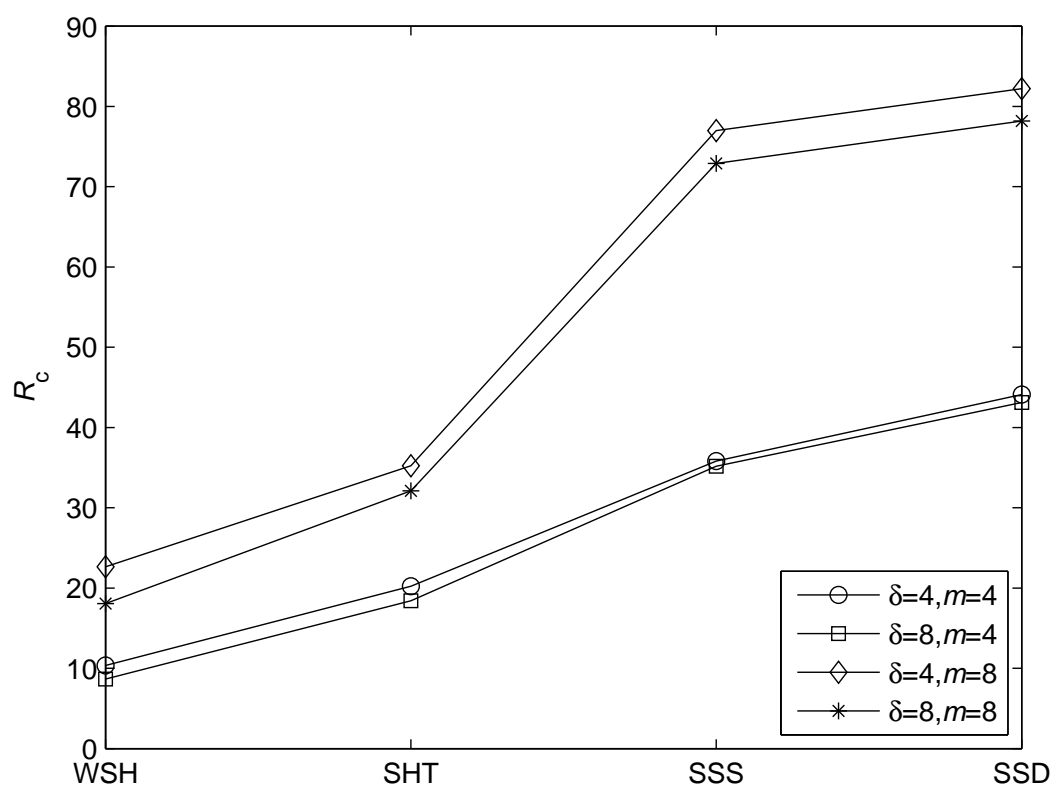

Figure 1: $R_{c}$ of different routing strategies. BBV network with $n=200$ and $w_{0}=1, C_{i}=1$

\section{Results and Discussion}

To obtain the critical packet generation rate $R_{c}$ in simulations, we use the order parameter[1]:

$$
\eta=\lim _{t \rightarrow \infty} \frac{\langle\Delta \Theta\rangle}{R \Delta t}
$$

where $\Delta \Theta=\Theta(t+\Delta t)-\Theta$, with $\langle\cdots\rangle$ indicating average over time windows of width $\Delta t$, and $\Theta(t)$ is the total number of packets in the network at time $t$. At the early stage, when $R$ is very small, the generated packets can be delivered, $\langle\Delta \Theta\rangle$ is less than zero and so is $\eta$. Where $\eta$ is greater than zero, we can obtain the critical packet generation rate $R_{c}$.

In figure 1 , we plot the critical packet generation rate $R_{c}$ of different routing strategies in a BBV network with $n=200$ and $\omega_{0}=1$. (For every network, 10 instances are generated and for each instance, we run 10 simulations. The results are the average over all the simulations.)

Fig.1 shows that when each node has the same packet delivery capability, the WSH routing strategy is the most sensible to traffic congestion. The SHT routing strategy is better than WSH, and the SSD routing strategy has the maximum transfer capacity. Moreover, the greater the new added edge number $\mathrm{m}$ is, the more efficient our routing strategies are. Take networks with $\delta=4, m=4$ and $\delta=4, m=8$ for example, the SHT, SSS and SSD routing strategies can enhance the critical packet generation rate $R_{c} 95.72 \%, 246.53 \%, 326.75 \%$ than the WSH routing strategy correspondingly in network with $\delta=4, m=4$ while $112.95 \%, 306.81 \%, 398.81 \%$ 

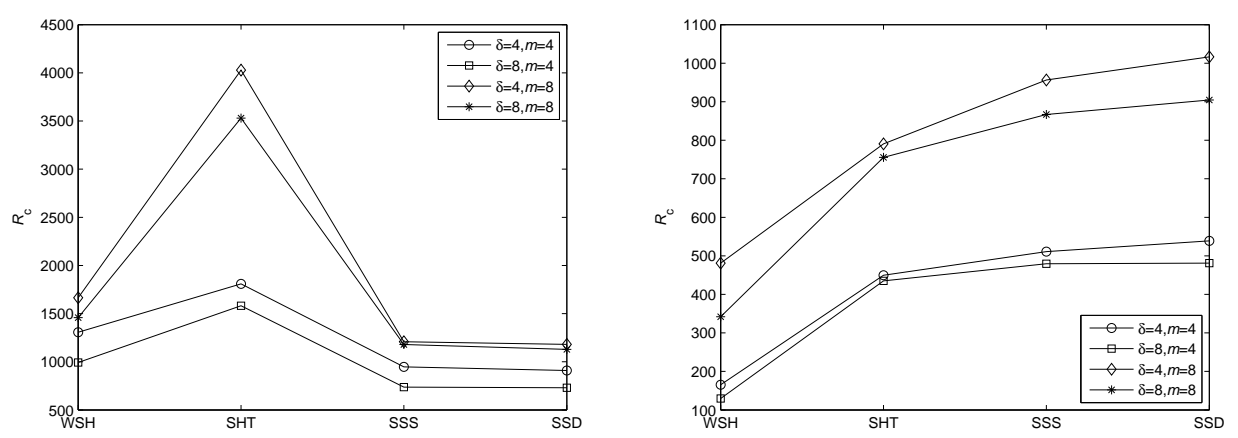

Figure 2: $R_{c}$ of different routing strategies. BBV network with $n=200$ and $w_{0}=1$ (a) $C_{i}=s_{i} /<s>$ (b) $C_{i}=k_{i} /<k>$
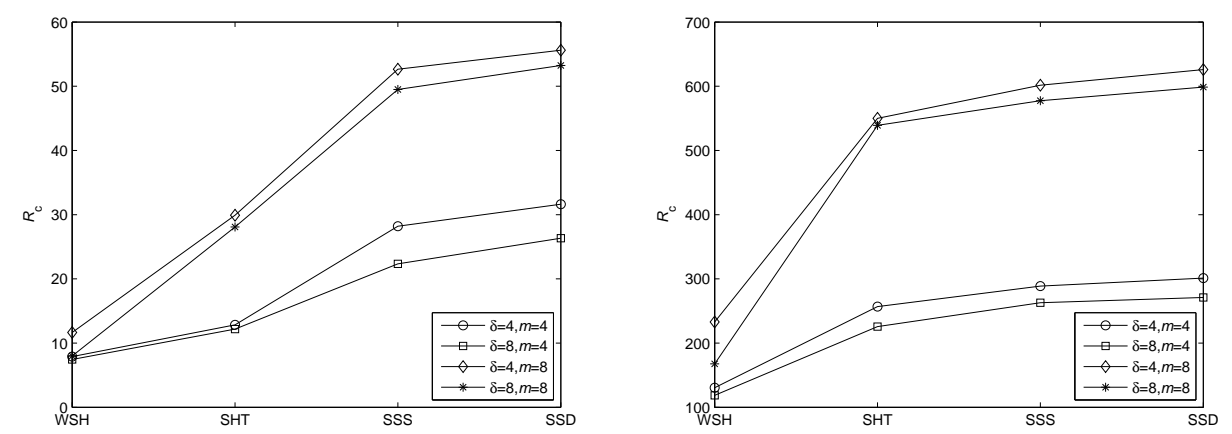

Figure 3: $R_{c}$ of different routing strategies. BBV network with $n=100$ and $w_{0}=1$ (a) $C_{i}=1$ (b) $C_{i}=k_{i} /<k>$

in network with $\delta=4, m=8$. When the new added edge number $m$ is increased, there are more edges in the network and there might be more traditional dijkstra shortest paths between nodes consequently. That is why our routing strategies are more efficient with larger parameter $m$.

Then we turn to the other two schemes: the node delivery capacity is proportional to the node strength $s_{i}$ and the node degree $k_{i}$. Simulation results are shown in Fig.2(a) and Fig.2(b) correspondingly.

Fig.2(a) presents that when the node delivery capacity is considered to be proportional to the node strength, the SHT routing strategy is the most effective while the SSD routing strategy has the largest $R_{c}$ when the node delivery capacity is considered to be proportional to the node degree as shown in Fig.2(b). Fig.2(a) shows that our routing strategies do not work well in STR scheme. In DEG scheme, the SHT routing strategy is also better than WSH, and the SSD routing strategy still has the maximum transfer capacity. However, the gap among the SHT and SSS and SSD routing strategies is narrowed. And our routing strategies are more efficient with the greater new added edge number.

Then we check the impact of the node number $n$ on our routing strategies. We test our routing strategies on BBV weighted networks with $n=100$ nodes to achieve the simulation results of CON scheme and DEG scheme as shown in Fig. 3.

Fig.3(a) and Fig.3(b) display the influence of node number our routing strategies. We can 

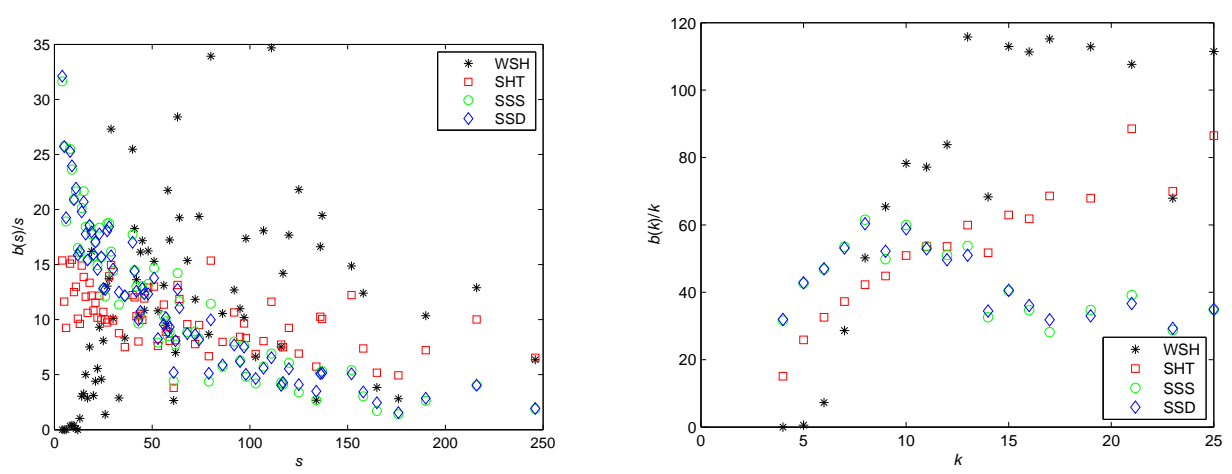

Figure 4: Betweenness per node. BBV network with $n=200, \delta=4, m=4$ and $w_{0}=1$ (a) $C_{i}=s_{i} /<s>$ (b) $C_{i}=k_{i} /<k>$

discover that the SSD route is still the best way to enhance the critical packet generation rate. And by comparing Fig.3(a) with Fig.1 and Fig.3(b) with Fig.2(b), we can discover that the node number $n$ has a little effect on the transfer capacity.

To achieve heuristic explanation for the routing strategies corresponding to the highest transfer capacity, we investigate the betweenness distribution on the network as presented in Fig.4.

The betweenness normalized by the strength of STR scheme is shown in Fig.4(a) and normalized by the degree of DEG scheme shown in Fig.4(b). In both figures, the load of the most effective routing strategy is distributed more evenly than the other three. In Fig.4(a), the betweenness divide by the strength of the SHT routing strategy is relatively flat which means the node with higher strength forward more packets. And in Fig.4(b), the node with higher degree forward more packets while using the SSD routing strategy. It is obvious that the traffic load under the SHT routing strategy for STR scheme and under the SSD routing strategy for DEG scheme are distributed evenly to the nodes according to their strength and degree correspondingly.

Those routing strategies which elongate the average path length $L_{A V E}$ unnecessarily may not be efficient for network communications. Thus it is of great importance for a routing strategy to maintain the small-world phenomenon, i.e. $L_{A V E} \propto \ln n$. In our routing strategies, all the paths are the shortest path between arbitrary nodes which means the small-world phenomenon is still maintained in our routing strategies.

Finally, we test our routing strategies for three schemes on real world network. We choose the USAir 97 network (network of direct flight connections between US airports for the year 1997, http://vlado.fmf.uni-lj.si/pub/networks/data/) with 332 nodes and 2126 edges. Simulation results are shown in Tab.2.

Table 2: $R_{c}$ of different routing strategies of USA airport network

\begin{tabular}{|c|c|c|c|c|}
\hline & WSH & SHT & SSS & SSD \\
\hline CON & 3.49 & 4.86 & 5.85 & 5.87 \\
\hline STR & 2.24 & 2.26 & 2.24 & 2.24 \\
\hline DEG & 83.69 & 161.96 & 169.03 & 169.90 \\
\hline
\end{tabular}

From Table 2 we can discover that in CON scheme, the SSD routing strategy has the maximum transfer capacity which is only a bit higher than the SSS routing strategy. The WSH 
routing strategy has the lowest transfer capacity. It means when each node has the same packet delivery capability, our SSS routing strategy and our SSD routing strategy can enhance the transfer capacity in real world network. And in STR scheme, the SHT routing strategy has the maximum transfer capacity which is also the same as the computer generated weighted network. When it turns to the DEG scheme, the SSS routing strategy and the SSD routing strategy also achieve better results than the traditional routing strategy. In a word, the SSS routing strategy and the SSD routing strategy also works well in the real world network in the CON scheme and in the DEG scheme.

\section{Conclusions}

Considering the different node delivery capability, this paper has proposed two novel routing strategies to enhance the network transfer capacity in weighted networks. The characteristic of our strategy is to specify the shortest path according to three kinds of different node delivery capability schemes. The simulation shows that when each node has the same packet delivery capability, we can select the path with the minimal number of nodes and with minimum sum of node degree. And this routing path is also optimal in the scheme which the node delivery capacity is considered to be proportional to the node degree. When the node delivery capacity is considered to be proportional to the node strength, our routing strategies do not work. It is worth mentioning that our routing strategies are more efficient with the more new added edge. At last, we apply our routing strategies on the USAir 97 network to show the validity of our routing strategies on real world network. Moreover, the above-mentioned research may throw lights on designing better communication protocols.

\section{Acknowledgment}

This work was partially supported by the National Natural Science Foundation of China (Grants No. 61373136 and 61375121), the Natural Science Foundation of Jiangsu Province, China (Grant No. BK2012082), the Research Foundation of Jinling Institute of Technology (Grant No. JIT-B-201406) and sponsored by Qing Lan Project. The author also gratefully acknowledges the helpful comments and suggestions of the reviewers, which have improved the presentation.

\section{Bibliography}

[1] Arenas A. et al (2001); Communication in networks with hierarchical branching, Physical Review Letters, ISSN 0031-9007. 86(14): 3196-3199.

[2] Guimera R. et al (2002); Optimal network topologies for local search with congestion, Physical Review Letters, ISSN 0031-9007, DOI://dx.doi.org/10.1103/PhysRevLett.89.248701.

[3] Zhang, G.Q. (2010); On cost-effective communication network designing. Europhysics Letters, ISSN 0295-5075. 89(3): 38003.

[4] Dijkstra E.W. (1959); A note on two problems in connexion with graphs. Numerische Mathematik, ISSN 0029-599X, 1(1): 269-271.

[5] Yan G. et al (2006); Efficient routing on complex networks. Physical Review E, ISSN 15393755. 73(4): 046108. 
[6] Wang W.X. et al. (2006); Traffic dynamics based on local routing protocol on a scale-free network. Physical Review E, ISSN 1539-3755, 73(2): 026111.

[7] Yin C.Y. et al (2006); Traffic dynamics based on an efficient routing strategy on scale free networks, The European Physical Journal B, ISSN 1434-6028. 49(2): 205-211.

[8] Toroczkai Z.; Bassler K.E. (2004); Network dynamics: Jamming is limited in scale-free systems. Nature, 428, 716 (15 April 2004), doi:10.1038/428716a.

[9] Wu Y.H. et al (2013); Performance Analysis of Epidemic Routing in Delay Tolerant Networks with Overlapping Communities and Selfish Nodes, International Journal of Computers Communications \& Control, ISSN 1841-9844, 8(5): 744-753.

[10] Newman M.E.J. (2001); Scientific collaboration networks. II. Shortest paths, weighted networks, and centrality. Physical Review E, ISSN 1063-651X. 64(1): 016132.

[11] Almaas E. et al (2004); Global organization of metabolic fluxes in the bacterium Escherichia coli, Nature, ISSN 0028-0836. 427(6977): 839-843.

[12] Barrat A. et al (2004); The architecture of complex weighted networks. PNAS, ISSN 00278424. 101(11): 3747-3752.

[13] Pastor-Satorras R.; Vespignani A. (2007); Evolution and structure of the Internet: A statistical physics approach, Cambridge University Press, ISBN 9780521714778.

[14] Brandes U. (2001); A faster algorithm for betweenness centrality. Journal of Mathematical Sociology, ISSN 0022-250X, 25(2): 163-177.

[15] Shao, F. (2013); Optimal Transport on Weighted Networks for Different Node Delivery Capability Schemes, The Scientific World Journal, http://dx.doi.org/10.1155/2013/378083.

[16] Barrat A. et al (2004); Modeling the evolution of weighted networks. Physical Review E, ISSN 1539-3755. 70(6): 066149.

[17] Barrat, A. et al (2004); Weighted evolving networks: coupling topology and weight dynamics. Physical Review Letters, ISSN 1079-7114. 92(22): 228701.

[18] Barthelemy M. et al (2005); Characterization and modeling of weighted networks. Physica A: Statistical Mechanics and its Applications, ISSN 0378-4371. 346(1-2): 34-43.

[19] Freeman L.C. (1977); A set of measures of centrality based on betweenness. Sociometry, ISSN 0038-0431, 40(1): 35-41. 